\title{
Investigating Obesity-related Factors for Childhood Asthma
}

\author{
Yang-Ching Chen ${ }^{1}$, Ming-Wei $\mathrm{Su}^{2}$, Ben Brumpton ${ }^{3}$, and Yungling Lee ${ }^{2}$ \\ ${ }^{1}$ Taipei Medical University \\ ${ }^{2}$ Institute of Biomedical Sciences Academia Sinica \\ ${ }^{3}$ Clinic for Thoracic and Occupational Medicine
}

September 25, 2021

\begin{abstract}
Background: We tested the hypothesis that multiple obesity-related risk factors (obesity, physical activity, cardiopulmonary physical fitness, sleep-disorder breathing (SDB), and sleep quality) are associated with childhood asthma using a Mendelian randomization (MR) design. Furthermore, we aim to investigate whether these risk factors were associated with incident asthma prospectively. Methods: In total, 7069 children aged 12 from the Taiwan Children Health Study were enrolled in the current study. Cross-sectional logistic regression, one-sample MR, summary-level MR sensitivity analyses, and prospective survival analyses were used to investigate each causal pathway. Results: In MR analysis, three of the five risk factors (obesity, SDB, and sleep quality) were associated with asthma, with the highest effect sizes per interquartile range (IQR) increase observed for sleep quality (odds ratio $[\mathrm{OR}]=1.42 ; 95 \%$ confidence interval $[\mathrm{CI}]: 1.06$ to 1.92$)$ and the lowest for obesity $(\mathrm{OR}=1.08 ; 95 \% \mathrm{CI}$ : 1.00-1.16). In the prospective survival analysis, obesity showed the highest risk of incident asthma per IQR increase (hazard ratio $[\mathrm{HR}]=1.28 ; 95 \% \mathrm{CI}: 1.05$ to 1.56$)$, followed by $\mathrm{SDB}(\mathrm{HR}=1.18 ; 95 \% \mathrm{CI}: 1.08$ to 1.29$)$ and sleep quality (HR $=1.10$; 95\% CI: 1.03 to 1.17). Conclusion: The most plausible risk factors for asthma were obesity, SDB, and poor sleep quality. For the prevention of childhood asthma, relevant stakeholders should prioritize improving children's sleep quality and preventing obesity comorbidities such as SDB.
\end{abstract}

\section{Investigating Obesity-related Factors for Childhood Asthma}

Yang-Ching Chen ${ }^{1,2,3,4}$, Ming-Wei Su ${ }^{5}$, Ben M. Brumpton ${ }^{6,7,8}$, Yungling L. Lee ${ }^{5,9}$

\section{Short running title: Risk factors for childhood asthma}

${ }^{1}$ Department of Family Medicine, Taipei Medical University Hospital, Taipei Medical University, Taipei, Taiwan

${ }^{2}$ Department of Family Medicine, School of Medicine, College of Medicine, Taipei Medical University, Taipei, Taiwan

${ }^{3}$ School of Nutrition and Health Sciences, College of Nutrition, Taipei Medical University, Taipei, Taiwan

${ }^{4}$ Graduate Institute of Metabolism and Obesity Sciences, Taipei Medical University, Taipei, Taiwan

${ }^{5}$ Institute of Biomedical Sciences, Academia Sinica, Taipei, Taiwan

${ }^{6}$ Clinic for Thoracic and Occupational Medicine, St. Olavs Hospital, Trondheim, Norway

${ }^{7}$ MRC Integrative Epidemiology Unit, University of Bristol, Bristol, United Kingdom

${ }^{8}$ K.G. Jebsen Center for Genetic Epidemiology, Norwegian University of Science and Technology, Trondheim, Norway

${ }^{9}$ College of Public Health, China Medical University, Taichung, Taiwan 


\section{Correspondence and requests for reprints should be addressed to}

Yungling Leo Lee, MD, PhD

Institute of Biomedical Sciences, Academia Sinica, 128 Academia Road, Sec. 2, Taipei 11529, Taiwan

Tel: +886-2-2652-3013

E-mail:leolee@ibms.sinica.edu.tw

Conflicts of interest: The authors declare no conflict of interest.

Financial disclosure: This work was supported by the National Health Research Institutes of Taiwan [NHRI-EX110-10718PC and NHRI-EX109-10606PI]. National Health Research Institutes supported genomic study expenditures in this study.

Word count: 227 for abstract; 2810 for text.

Abbreviations : BMI, body mass index; CI, confidence interval; DTHM, discrete-time hazard model; Fe NO$_{\text {, }}$ fractional exhaled nitric oxide; FEV1/FVC, forced expiratory volume in 1 second/forced vital capacity; IV, instrumental variable; MR, Mendelian randomization; OR, odds ratio; GRS, genetic risk score; SDB, sleepdisordered breathing; SNP, single-nucleotide polymorphism; TCHS, Taiwan Children Health Study; 2SLS, two-stage least square

Author contributions: Dr. Yang-Ching Chen contributed to data collection, hypothesis generation, data interpretation, and writing the manuscript. Prof. Yungling Lee reviewed the study design and data collection and interpretation, supervised the study, and critically revised the manuscript for essential intellectual content. Dr. Ming-Wei Su and Prof. Ben Brumpton assisted in a critical part of statistical analysis and the manuscript review. All the authors approved the final manuscript for submission and publication and agreed to be accountable for all aspects of the work.

\section{ABSTRACT}

Background: We tested the hypothesis that multiple obesity-related risk factors (obesity, physical activity, cardiopulmonary physical fitness, sleep-disorder breathing (SDB), and sleep quality) are associated with childhood asthma using a Mendelian randomization (MR) design. Furthermore, we aim to investigate whether these risk factors were associated with incident asthma prospectively.

Methods: In total, 7069 children aged 12 from the Taiwan Children Health Study were enrolled in the current study. Cross-sectional logistic regression, one-sample MR, summary-level MR sensitivity analyses, and prospective survival analyses were used to investigate each causal pathway.

Results: In MR analysis, three of the five risk factors (obesity, SDB, and sleep quality) were associated with asthma, with the highest effect sizes per interquartile range (IQR) increase observed for sleep quality (odds ratio $[\mathrm{OR}]=1.42 ; 95 \%$ confidence interval $[\mathrm{CI}]: 1.06$ to 1.92$)$ and the lowest for obesity $(\mathrm{OR}=1.08$; 95\% CI: 1.00-1.16). In the prospective survival analysis, obesity showed the highest risk of incident asthma per IQR increase (hazard ratio $[\mathrm{HR}]=1.28 ; 95 \%$ CI: 1.05 to 1.56 ), followed by SDB (HR $=1.18 ; 95 \%$ CI: 1.08 to 1.29 ) and sleep quality ( $\mathrm{HR}=1.10 ; 95 \% \mathrm{CI}: 1.03$ to 1.17 ).

Conclusion: The most plausible risk factors for asthma were obesity, SDB, and poor sleep quality. For the prevention of childhood asthma, relevant stakeholders should prioritize improving children's sleep quality and preventing obesity comorbidities such as SDB.

Keywords: Obesity; Asthma; Mendelian randomization study; Prospective study; Pulmonary function

\section{INTRODUCTION}

Childhood obesity and asthma have posed a parallel epidemic over the past three decades ${ }^{1}$. According to a meta-analysis of 6 prospective children studies, obesity was significantly associated with 2 -fold times increased risk for incident asthma ${ }^{2}$. Obese asthma is a complex syndrome, including different phenotypes 
of diseases that warrant further investigations. Hence, identifying obesity-related risk factors behind this causal relationship is essential to inform preventive interventions. The obesity-related risk factors could be composed of hereditary factors and lifestyle factors, such as physical inactivity and inadequate sleep.

Multiple obesity-related factors may potentially contribute to the link of childhood asthma; for example, physical activity or physical fitness levels, poor sleep quality, and some obesity comorbidities, such as sleepdisordered breathing (SDB), may also play a role. We previously performed a bidirectional MR analysis, with the results suggesting that adiposity antedates asthma ${ }^{3}$. Moreover, the causal relationships between physical activity or physical fitness levels and asthma have long been debated. Some studies have supported the causal relationship between physical fitness and asthma incidence in prospective studies ${ }^{4,5}$, whereas others have reported that physical fitness does not influence asthma directly ${ }^{6}$. Moreover, poor sleep quality and SDB are common in children with asthma. Prospective studies of insomnia and new-onset asthma have been conducted in adults ${ }^{7,8}$ but not in children. Using two-sample MR analysis, Dashti et al. reported a U-shaped association between sleep duration and asthma in an adult study ${ }^{9}$. Although many studies have reported the obesity-related factors leading to asthma, accurately appraising the causality of these factors in asthma is essential for future preventive interventions.

Epidemiological studies have identified some obesity-related factors associated with asthma, but these associations can be subject to confounding and reverse causations. Mendelian randomization (MR) can be used to strengthen causal inference indicated by observational studies by using genetic variants as instrumental variables for risk factors. A genetic risk score (GRS) for an individual obesity-related factor can be regarded as a genetic proxy for this risk factor. Therefore, if a certain obesity-related factor is causally related to asthma, a GRS for this risk factor should also be associated with asthma.

In this study, we addressed the aforementioned limitations of traditional observational methods and focused on several obesity-related factors (obesity, physical activity, cardiopulmonary physical fitness, SDB, and sleep quality) in which candidate genetic instruments could be defined. We aimed to (1) elucidate the causality of multiple obesity-related factors and childhood asthma by using MR, (2) conduct prospective analysis to strengthen the temporal relationship between these risk factors and asthma, and (3) describe the inherited susceptibility of each factor on asthma from representative GRSs.

\section{MATERIALS AND METHODS}

\section{Study participants}

We obtained observational data on 7069 children aged 12 from the Taiwan Children Health Study (TCHS). The TCHS was a nationwide prospective study that comprised two cohorts of children, with $8.64 \%$ of the participants having physician-diagnosed asthma. The analysis herein included 3233 children in Cohort 1 and 3836 children in Cohort 2. The detailed study design and recruitment strategy were previously described ${ }^{10}$. Follow-up surveys were performed in 2012 and 2018 until these children were 17 years old. This study was approved by the Ethics Committees and was conducted according to the Declaration of Helsinki after obtaining informed consent from participating parents and children.

\section{Definition of respiratory outcomes}

We used answers from the parents to determine whether a child had physician-diagnosed asthma. The question was, "Has a doctor ever diagnosed your child as having asthma?" In the prospective survival analysis, incident asthma was calculated by excluding asthma history before the age of 11 years. Incident asthma was determined at age 12 and 17 years by parents' affirmative answer to whether their child had been physician-diagnosed as having asthma during the follow-up period. The atopic status of asthma was determined by measuring the fractional exhaled nitric oxide $\left(\mathrm{Fe}_{\mathrm{NO}}\right.$ ) by using a portable NO analyzer (NIOX MINO Airway Inflammation Monitor; Aerocrine AB, Solna, Sweden). We categorized children with asthma into the atopic or nonatopic group by using $20 \mathrm{ppb}$ of $\mathrm{Fe}_{\mathrm{NO}}$ as the cutoff value. ${ }^{11}$

A pulmonary function test was performed in accordance with a previously standardized protocol ${ }^{12}$ at age 12 years. The forced expiratory volume in 1 second (FEV1)/forced vital capacity (FVC) ratio, which is a 
marker of obstructive type pulmonary function impairment, was also used as a respiratory outcome.

\section{Measurement of multiple obesity-related factors}

Five obesity-related risk factors were investigated at age 11-12, where suitable genetic instruments were available for inclusion in the analysis: obesity, physical activity, cardiopulmonary physical fitness, SDB, and sleep quality.

Physical activity was assessed using a questionnaire and evaluation at school. We used the Chinese version of the International Physical Activity Questionnaire ${ }^{13}$ to determine a child's physical activity levels. Participants were asked a series of questions pertaining to intensity, duration, and frequency of physical activity in the preceding week. Physical activities were categorized into vigorous, moderate, and mild, according to their metabolic equivalent (MET) values, namely $>6$ MET, 3-6 MET, and $<3$ MET. Physical activities were then quantified according to their average MET expenditure per day ${ }^{14}$. Cardiorespiratory physical fitness was assessed through an 800-m sprint, a surrogate marker of predicted maximal oxygen consumption ${ }^{15}$, during school visits by using a standardized protocol ${ }^{16}$. It was first converted into $z$ scores by using sex- and age-specific means and standard deviations. Hence, a higher cardiorespiratory physical fitness index signifies greater physical fitness.

SDB was determined by using a Chinese version of the Children's Sleep Habit Questionnaire, which was completed by the parent. Researchers compared this questionnaire with objective polysomnography-defined obstructive sleep apnea and found it to be a valid tool for detecting the presence of SDB ${ }^{17}$. Sleep quality was evaluated through the Chinese version of the Pittsburgh Sleep Quality Index (PSQI) questionnaire. The questionnaire consists of 19 self-rated questions that are grouped into seven subscale components: sleep quality, sleep latency, sleep duration, habitual sleep efficiency, daytime dysfunction, sleep disturbances, and use of sleep medications ${ }^{18}$. Each component score has equal weight and is measured on a Likert scale of 0 (no difficulty) to 3 (severe difficulty). The seven component scores are summed to yield a global PSQI score, with a range of 0-21; higher scores indicate poorer sleep quality.

We used body mass index (BMI) as a marker of obesity and calculated it by dividing body weight in kilograms by height in meters squared $\left(\mathrm{kg} / \mathrm{m}^{2}\right)$. Subsequently, BMI was converted into age- and sex-specific $z$ scores (z-BMI) according to the World Health Organization growth standards for school-aged children and adolescents $^{19}$.

\section{MR analysis}

We performed $\mathrm{MR}^{20}$ analyses by using genetic instrumental variables (IVs) to proxy for candidate obesityrelated factors. The use of MR enables unbiased causal effects of risk factors on respiratory outcomes to be estimated if the following assumptions are met: (1) the genetic IVs are robustly associated with the risk factor of interest; (2) the genetic IVs are not associated with any confounding factors; (3) the pleiotropic pathways through which the IVs influence the outcome are all due to the risk factors. GRS composed of risk factors associated-single-nucleotide polymorphism (SNP) was used as IVs. The dosage of the effect allele was multiplied by the regression coefficients of each SNP on associated traits divided by the mean value of all regression coefficients.

By referencing candidate SNPs from large genome-wide association studies, we collected well-known genetic variants associated with the five obesity-related factors. To avoid the pleiotropic effects of genetic variants, SNPs previously reported to be associated with asthma and confounders were excluded. After we selected SNPs for the obesity-related factors, some criteria were applied to filter the selected SNPs for quality control: (1) minor allele frequency of [?]5\%, (2) genotyping call rate of $>98 \%$ for all children, and (3) no linkage disequilibrium with candidate SNPs. The primers were designed using the National Genotyping Center of the Academia Sinica platform (http://lims.ngc.sinica.edu.tw/service/) ${ }^{21}$. Genotyping was performed using Sequenom iPLEX matrix-assisted laser desorption/ionization-time of flight mass spectrometry at the National Center for Genome Medicine, Taiwan. The online supplementary file provides details on how candidate SNPs were selected. 


\section{Statistical analysis}

We initially conducted cross-sectional logistic models in the observational models (Figure 1). To investigate whether a factor is a causal factor for asthma, we conducted MR two-stage least squares (2SLS) and MR sensitivity analyses for all relationships. MR 2SLS analysis is a fundamental method in MR for both binary and continuous variables of exposure and outcomes ${ }^{22}$. MR sensitivity analyses (MR inverse-variance weighted, Egger [slope], and weighted median method) were used to support the validity of the causal inference from the MR analysis ${ }^{23}$. To elucidate whether various factors predict incident asthma in later life, we also applied a discrete-time hazard model (DTHM). The DTHM is a survival analysis model that enables the estimation of hazard ratios (HRs) and 95\% confidence intervals (CIs) when an event occurrence may be considered in a discrete period; baseline information on these factors was collected on disease-free individuals at age 11, and the outcomes were incident asthma at ages 12 and 17. The observational models and DTHM were adjusted for several potential confounders, such as age, sex, parental education, and family income. Odds ratios (ORs), HRs, and beta values were estimated using the IQR increase of the factors.

The GRS for a risk factor can be considered a genetic proxy. To compare the effect of these factors on asthma, as identified from observational and MR analyses, we further utilized the GRS to calculate the predicted prevalence of asthma. We computed the sample mean per quintile for each of the GRS to serve as the new dataset for prediction by logistic regression. To estimate the predicted probability of the GRS of an obesity-related factor, we included the means per quintile for asthma. All model regression and sensitivity analyses were performed using R software and R package (ivreg) MR 2SLS analysis (version 3.3.2, RStudio, Inc., Boston, MA, USA).

\section{RESULTS}

In the present study, the TCHS population comprised 7069 children at age 12 (Table 1), and 4328 children received follow-up surveys at age 17 (Table E1). Among them, 253 (7.83\%) in Cohort 1 and 358 (9.33\%) in Cohort 2 were children with physician-diagnosed asthma.

\section{Observational and MR association}

By using observational and MR 2SLS analysis, we calculated the causal associations of multiple obesityrelated factors with asthma and pulmonary function (Table 2). The F statistics for obesity, physical activity, cardiopulmonary physical fitness, sleep-disordered breathing, and sleep quality were 80.60, 18.62, 11.68, 10.27, and 7.09, respectively. Three of the five obesity-related factors exhibited robust associations with asthma in both the observational and MR analysis. In order of decreasing effect sizes in MR analysis, these were sleep quality, SDB, and obesity, with the highest effect sizes per IQR increase, observed for sleep quality $(\mathrm{OR}=1.42 ; 95 \% \mathrm{CI}, 1.06$ to 1.92$)$ and the lowest for obesity ( $\mathrm{OR}=1.08 ; 95 \% \mathrm{CI}, 1.00$ to 1.16$)$. Moreover, a one-IQR increase in z-BMI was also associated with a decrease in FEV1/FVC $(\beta=-2.44,95 \% \mathrm{CI},-4.24$ to -0.65). Most of the MR sensitivity analysis results for the associations between obesity and respiratory outcomes were consistent with the main result (Table E2).

The GRSs of four tested factors (except for sleep quality) were robustly correlated with each factor and were associated with asthma (Table E3). Tables E4-8 reveal the associations of SNPs with the factors and asthma. The data listed in Tables E9-13 verify that most SNPs were independent of confounders.

\section{Prospective survival analysis}

Table 3 presents the analysis of the five obesity-related factors for incident asthma at ages 12 and 17 years in the survival model. Three of the five factors were associated with increased incident asthma risk. Among the three risk factors, obesity presented the highest risk of incident asthma (HR: 1.28, 95\% CI: 1.05 to 1.56), followed by a 1.18-, and 1.10-fold higher risk of incident asthma per IQR increase for SDB (HR: 1.18, 95\% CI: 1.08 to 1.29 ), and sleep quality (HR: $1.10,95 \%$ CI: 1.03 to 1.17 ), respectively. In the subgroup analysis, SDB was associated with an increase in atopic asthma (HR: 1.50, 95\% CI: 1.22 to 1.84 ).

\section{Genetic risk score-predicted risks}


Figure 2 displays the inherited susceptibility of each risk factor on asthma from representative GRSs. We demonstrated the predicted prevalence of asthma for the three risk factors (obesity, SDB, and sleep quality) corresponding to the mean GRS in each quintile. All three factors exhibited an increasing trend of predicted prevalence of asthma following the increase in quintile. To illustrate, the predicted prevalence of asthma was $6 \%$ for those whose GRS for SDB and sleep quality was in the lowest $20 \%$ of the sample (Q1) compared with $13 \%$ for those whose GRS was in the highest $20 \%$ (Q5). However, obesity revealed a less prominent increase. The predicted prevalence of asthma for those whose GRS for obesity in Q1 was 7\%, with an increasing trend to $11 \%$ for those with scores in Q5.

\section{DISCUSSION}

Among the five obesity-related factors examined for causality with childhood asthma, we obtained consistent evidence that obesity, SDB, and sleep quality are observationally and genetically associated with asthma. Prospective survival analysis also confirmed the temporal relationship. When stratified by asthma subphenotypes, SDB was associated with atopic incident asthma. Detection and treatment of sleep-related symptoms may reduce the risk of asthma.

In our data, poor sleep quality and SDB were the strongest risk factors contributing to asthma in the observational and MR analyses. Their predicted prevalence of asthma was also the highest in the Q5 GRS. Two previous prospective studies support our findings that insomnia increases the risk of asthma in adults ${ }^{7,8}$. Brumpton et al. reported an increased risk of asthma in those having problems initiating sleep, waking up too early, and experiencing nonrestorative sleep ${ }^{8}$. Moreover, Lin et al. conducted a population-based study in Taiwan and found that patients with insomnia had a 2-fold risk of having new-onset asthma during the 4-year follow-up ${ }^{7}$. On the other hand, our study is the first to investigate this association among school-age children. Although the prevalence of insomnia among school-age children is not high, the global pandemic of sleep loss among children has gained the attention of public health and school officials ${ }^{24}$. According to a large-scale meta-analysis ${ }^{25}$, European adolescents sleep for an average of 500 minutes per day, whereas Asian adolescents sleep for only 400 minutes per day on weekdays. In our study, we used the PSQI questionnaire, representing a combination of various components of sleep parameters, to assess sleep quality in children. Poor sleep quality was a robust risk factor for asthma in the three models. The mechanism behind poor sleep quality leading to asthma may be explained by the shifting of $\mathrm{T}$ helper (Th) cell activity toward a Th2 cytokine profile under chronic sleep deprivation ${ }^{26}$. Moreover, protracted upregulation of inflammatory markers, such as increased interleukin (IL)-6, was also observed among patients with poor sleep ${ }^{27}$. IL-6 production induced by poor sleep quality might exacerbate airway hypersensitivity. Other potential mechanisms, such as poor sleep-induced chronic inflammatory status, manifesting as increased NF-kB level ${ }^{28}$ and decreased IFN- ${ }^{29}$, may also explain why poor sleep quality triggers new-onset asthma.

SDB was another strong risk factor for asthma in the present study. Although researchers have postulated that SDB and asthma exhibit similar airway inflammation, the temporal relationship between the two remains unclear $^{30}$. The current study is the first to confirm the causal association using both MR analysis and a prospective cohort. SDB is associated with excessive daytime sleepiness, the release of proinflammatory cytokines, and increased leptin level, contributing to the development of asthma ${ }^{31}$. Our finding that SDB predicts atopic incident asthma is supported by one study ${ }^{32}$ but contradicted by another related study, which found that SDB is associated with neutrophilic airway inflammation ${ }^{33}$. Further studies are warranted to explain relevant mechanisms linking SDB and asthma.

The strength of the current analysis centers on the use of three methods (observational, MR, prospective) to investigate the causal associations between risk factors and asthma. Our review of the literature suggests that no related study has employed three methods (observational, MR, and survival analysis) to investigate modifiable obesity-related factors for childhood asthma. The unique, population-based children cohort with prospective follow-up surveys enabled this analysis. Our study used self-reported physical activity and sleep quality, which represents a potential limitation. However, the TCHS employed globally used validated questionnaires to assess physical activity and sleep quality, limiting the reporting bias. Moreover, F-statistic suggests that the GRS of sleep quality is a weak instrument, and we might be underpowered to detect 
small-moderate effect sizes, as compared to large sample GWAS studies.

\section{CONCLUSION}

We found that obesity, SDB, and sleep quality were consistently associated with asthma in observational, $\mathrm{MR}$, and prospective survival analysis models. After a series of complementary analyses to further strengthen the causal inference, poor sleep quality and SDB emerged as the most plausible causal risk factor, followed by obesity. Hence, lifestyle interventions aimed at promoting high-quality sleep and reducing SDB symptoms, such as weight management, might effectively reduce asthma risk.

\section{Acknowledgements:}

The authors thank the field workers, teachers, and other school staff who supported data collection, and all parents and children who participated in this study. We thank the National Health Research Institutes of Taiwan for grant support of this study [NHRI-EX110-10718PC and NHRI-EX109-10606PI]. We also thank the National Center for Genome Medicine (NCGM) in Taiwan for providing technical support for the genotyping.

\section{REFERENCE}

1. Lai L, Zhang T, Zeng X, Tan W, Cai L, Chen Y. Association between Physician-Diagnosed Asthma and Weight Status among Chinese Children: The Roles of Lifestyle Factors. International journal of environmental research and public health . 2020;17(5).

2. Chen YC, Dong GH, Lin KC, Lee YL. Gender difference of childhood overweight and obesity in predicting the risk of incident asthma: a systematic review and meta-analysis. Obes Rev . 2013;14(3):222-231.

3. Chen Y-C, Fan H-Y, Huang Y-T, Huang S-Y, Liou T-H, Lee YL. Causal relationships between adiposity and childhood asthma: bi-directional Mendelian Randomization analysis. International journal of obesity (2005) . 2019;43(1):73-81.

4. Hancox RJ, Rasmussen F. Does physical fitness enhance lung function in children and young adults? European Respiratory Journal . 2018;51(2):02.

5. Rasmussen F, Lambrechtsen J, Siersted HC, Hansen HS, Hansen NC. Low physical fitness in childhood is associated with the development of asthma in young adulthood: the Odense schoolchild study. European Respiratory Journal . 2000;16(5):866-870.

6. Chen YC, Tu YK, Huang KC, Chen PC, Chu DC, Lee YL. Pathway from central obesity to childhood asthma. Physical fitness and sedentary time are leading factors. Am J Respir Crit Care Med . 2014;189(10):1194-1203.

7. Lin Y-C, Lai C-C, Chien C-C, Chen C-M, Chiang S-R, Ho C-H, et al. Is insomnia a risk factor for new-onset asthma? A population-based study in Taiwan. BMJ open . 2017;7(11):e018714.

8. Brumpton B, Mai X-M, Langhammer A, Laugsand LE, Janszky I, Strand LB. Prospective study of insomnia and incident asthma in adults: the HUNT study. The European respiratory journal . 2017;49(2).

9. Dashti HS, Redline S, Saxena R. Polygenic risk score identifies associations between sleep duration and diseases determined from an electronic medical record biobank. Sleep . 2019;42(3).

10. Chen YC, Chen PC, Hsieh WS, Portnov BA, Chen YA, Lee YL. Environmental factors associated with overweight and obesity in taiwanese children. Paediatr Perinat Epidemiol . 2012;26(6):561-571.

11. Han YY, Forno E, Celedon JC. Adiposity, fractional exhaled nitric oxide, and asthma in U.S. children. Am J Respir Crit Care Med . 2014;190(1):32-39.

12. Lee YL, Hwang BF, Chen YA, Chen JM, Wu YF. Pulmonary function and incident bronchitis and asthma in children: a community-based prospective cohort study. PLoS One . 2012;7(3):e32477. 
13. Macfarlane DJ, Lee CC, Ho EY, Chan KL, Chan DT. Reliability and validity of the Chinese version of IPAQ (short, last 7 days). J Sci Med Sport . 2007;10(1):45-51.

14. Dogra S, Baker J, Ardern CI. The role of physical activity and body mass index in the health care use of adults with asthma. Ann Allergy Asthma Immunol . 2009;102(6):462-468.

15. Sandford GN, Allen SV, Kilding AE, Ross A, Laursen PB. Anaerobic Speed Reserve: A Key Component of Elite Male 800-m Running. International journal of sports physiology and performance . 2019;14(4):501508.

16. Education. Mo. Nationwide Physical Fitness Survey of School Children and Youth. . Ministry of Education, Taipei . 2008.

17. Li AM, Cheung A, Chan D, Wong E, Ho C, Lau J, et al. Validation of a questionnaire instrument for prediction of obstructive sleep apnea in Hong Kong Chinese children. Pediatr Pulmonol . 2006;41(12):11531160.

18. Ho RT, Fong TC. Factor structure of the Chinese version of the Pittsburgh sleep quality index in breast cancer patients. Sleep Medicine . 2014;15(5):565-569.

19. WHO Multicenter Growth Reference Study Group. WHO child growth standards:length/height-for-age, weight-for-age, weight-for-length, weight-for height and body mass index-for-age: methods and development.Available at: https://www.who.int/tools/child-growth-standards 2021.

20. Davies NM, Holmes MV, Davey Smith G. Reading Mendelian randomisation studies: a guide, glossary, and checklist for clinicians. BMJ . 2018;362:k601.

21. Liu YL, Wang SC, Hwu HG, Fann CS, Yang UC, Yang WC, et al. Haplotypes of the D-Amino Acid Oxidase Gene Are Significantly Associated with Schizophrenia and Its Neurocognitive Deficits. PLoS One . 2016;11(3):e0150435.

22. Palmer TM, Sterne JA, Harbord RM, Lawlor DA, Sheehan NA, Meng S, et al. Instrumental variable estimation of causal risk ratios and causal odds ratios in Mendelian randomization analyses. Am J Epidemiol . 2011;173(12):1392-1403.

23. Burgess Sp, Bowden Jp, Fall Tp, Ingelsson Ep, Thompson SGp. Sensitivity Analyses for Robust Causal Inference from Mendelian Randomization Analyses with Multiple Genetic Variants.Epidemiology . 2017;28(1):30-42.

24. Matricciani L, Olds T, Petkov J. In search of lost sleep: secular trends in the sleep time of school-aged children and adolescents. Sleep Medicine Reviews . 2012;16(3):203-211.

25. Olds T, Blunden S, Petkov J, Forchino F. The relationships between sex, age, geography and time in bed in adolescents: a meta-analysis of data from 23 countries. Sleep Medicine Reviews . 2010;14(6):371-378.

26. Axelsson J, Rehman J-u, Akerstedt T, Ekman R, Miller GE, Höglund CO, et al. Effects of sustained sleep restriction on mitogen-stimulated cytokines, chemokines and T helper $1 / \mathrm{T}$ helper 2 balance in humans.PloS one . 2013;8(12):e82291.

27. Burgos I, Richter L, Klein T, Fiebich B, Feige B, Lieb K, et al. Increased nocturnal interleukin-6 excretion in patients with primary insomnia: a pilot study. Brain, behavior, and immunity . 2006;20(3):246-253.

28. Tak PP, Firestein GS. NF-kappaB: a key role in inflammatory diseases. The Journal of clinical investigation . 2001;107(1):7-11.

29. Mitchell C, Provost K, Niu N, Homer R, Cohn L. IFN- $\gamma$ acts on the airway epithelium to inhibit local and systemic pathology in allergic airway disease. Journal of immunology (Baltimore, Md : 1950) . 2011;187(7):3815-3820. 
30. Castro-Rodriguez JA, Brockmann PE, Marcus CL. Relation between asthma and sleep disordered breathing in children: is the association causal? Paediatric respiratory reviews . 2017;22:72-75.

31. Tsaoussoglou M, Bixler EO, Calhoun S, Chrousos GP, Sauder K, Vgontzas AN. Sleep-disordered breathing in obese children is associated with prevalent excessive daytime sleepiness, inflammation, and metabolic abnormalities. The Journal of clinical endocrinology and metabolism . 2010;95(1):143-150.

32. Verhulst SL, Aerts L, Jacobs S, Schrauwen N, Haentjens D, Claes R, et al. Sleep-disordered breathing, obesity, and airway inflammation in children and adolescents. Chest . 2008;134(6):1169-1175.

33. Teodorescu M, Broytman O, Curran-Everett D, Sorkness RL, Crisafi G, Bleecker ER, et al. Obstructive Sleep Apnea Risk, Asthma Burden, and Lower Airway Inflammation in Adults in the Severe Asthma Research Program (SARP) II. The journal of allergy and clinical immunology In practice . 2015;3(4):566.

Figure Legends

Figure 1. Schematic drawing of analytical strategy

Figure 2. Predicted prevalence of asthma for the three obesity-related factors

Figure 2 footnote: GRS, genetic risk score.

Table 1. Characteristics of participants aged 12 years in the Taiwan Children Health Study

\begin{tabular}{|c|c|c|c|c|c|c|c|}
\hline Characteristics & Total & Total & Cohort 1 & Cohort 1 & Cohort 2 & Cohort 2 & $P$ value \\
\hline $\mathbf{N}$ & 7069 & 7069 & 3233 & 3233 & 3836 & 3836 & \\
\hline Age (years) & 12.1 & 0.67 & 12.70 & 0.34 & 11.66 & 0.48 & $<0.001$ \\
\hline Male sex & 3618 & $51.2 \%$ & 1669 & $51.6 \%$ & 1949 & $50.8 \%$ & 0.51 \\
\hline BMI $\left(\mathrm{kg} / \mathrm{m}^{2}\right)$ & 20.5 & 4.14 & 20.57 & 4.23 & 20.37 & 4.06 & 0.08 \\
\hline Parental education & & & & & & & $<0.001$ \\
\hline High school or below & 4110 & $58.1 \%$ & 2085 & $64.5 \%$ & 2025 & $52.8 \%$ & \\
\hline College or university & 2520 & $35.7 \%$ & 1026 & $31.7 \%$ & 1494 & $39.0 \%$ & \\
\hline Postgraduate & 439 & $6.21 \%$ & 122 & $3.77 \%$ & 317 & $8.26 \%$ & \\
\hline Family income $(\mathrm{NT} \$)$ & & & & & & & $<0.001$ \\
\hline$<600,000$ & 3926 & $55.5 \%$ & 2021 & $62.5 \%$ & 1905 & $49.7 \%$ & \\
\hline $600,001-1,000,000$ & 2143 & $30.3 \%$ & 848 & $26.2 \%$ & 1295 & $33.8 \%$ & \\
\hline$>1,000,001$ & 1000 & $14.2 \%$ & 364 & $11.3 \%$ & 636 & $16.6 \%$ & \\
\hline Physician-diagnosed asthma & 611 & $8.64 \%$ & 253 & $7.83 \%$ & 358 & $9.33 \%$ & 0.03 \\
\hline FEV1/FVC (\%) & 91.5 & 6.07 & 90.85 & 5.31 & 92.11 & 6.69 & $<0.001$ \\
\hline
\end{tabular}

BMI, body mass index; FEV1/FVC, forced expiratory volume in 1 s/forced vital capacity; NT\$, new Taiwan dollars.

All data are presented as means \pm standard deviation or numbers $(\%)$.

The number of participants does not add up to the total number because of missing data.

$\S \mathrm{P}$ value indicates the difference between the two cohorts.

Table 2. Observational and MR analysis of obesity-related factors in relation to asthma at age 12

\begin{tabular}{|c|c|c|c|c|c|c|c|}
\hline $\begin{array}{l}\text { Risk } \\
\text { factors }\end{array}$ & Category & $\begin{array}{l}\text { Physician- } \\
\text { diagnosed } \\
\text { asthma }\end{array}$ & $\begin{array}{l}\text { Physician- } \\
\text { diagnosed } \\
\text { asthma }\end{array}$ & $\begin{array}{l}\text { Physician- } \\
\text { diagnosed } \\
\text { asthma }\end{array}$ & $\begin{array}{l}F E V 1 / F V C \\
(\%)\end{array}$ & $\begin{array}{l}F E V 1 / F V C \\
(\%)\end{array}$ & $\begin{array}{l}F E V 1 / F V C \\
(\%)\end{array}$ \\
\hline & & OR & $95 \% \mathrm{CI}$ & $95 \% \mathrm{CI}$ & $\beta$ & $95 \% \mathrm{CI}$ & $95 \%$ CI \\
\hline
\end{tabular}




\begin{tabular}{|c|c|c|c|c|c|c|c|}
\hline $\begin{array}{l}\text { Risk } \\
\text { factors }\end{array}$ & Category & $\begin{array}{l}\text { Physician- } \\
\text { diagnosed } \\
\text { asthma }\end{array}$ & $\begin{array}{l}\text { Physician- } \\
\text { diagnosed } \\
\text { asthma }\end{array}$ & $\begin{array}{l}\text { Physician- } \\
\text { diagnosed } \\
\text { asthma }\end{array}$ & $\begin{array}{l}F E V 1 / F V C \\
(\%)\end{array}$ & $\begin{array}{l}F E V 1 / F V C \\
(\%)\end{array}$ & $\begin{array}{l}F E V 1 / F V C \\
(\%)\end{array}$ \\
\hline Obesity & \multicolumn{2}{|c|}{ Observational1.26 } & 1.07 & 1.47 & -1.16 & -1.37 & -0.95 \\
\hline$(28$ & MR & 1.08 & 1.00 & 1.16 & -2.44 & -4.24 & -0.65 \\
\hline $\begin{array}{l}\text { SNPs, F } \\
=80.60)\end{array}$ & \multicolumn{7}{|l|}{ 2SLS } \\
\hline $\begin{array}{l}\text { Physical } \\
\text { activity }\end{array}$ & \multicolumn{2}{|c|}{ Observationall.01 } & 1.00 & 1.01 & -0.09 & -0.24 & 0.06 \\
\hline $\begin{array}{l}(15 \\
\text { SNPs F }\end{array}$ & $\begin{array}{l}\text { MR } \\
\text { 2SIS }\end{array}$ & 0.91 & 0.82 & 1.00 & 0.77 & -1.39 & 2.93 \\
\hline $\begin{array}{l}\text { SNPs, F } \\
=18.62)\end{array}$ & \multicolumn{2}{|l|}{ 2SLS } & & & & & \\
\hline \multicolumn{3}{|c|}{$\begin{array}{l}\text { Cardiopulmonabservational0.99 } \\
\text { physical } \\
\text { fitness }\end{array}$} & 0.98 & 1.01 & 0.74 & 0.37 & 1.11 \\
\hline $\begin{array}{l}\text { nine } \\
(13\end{array}$ & MR & 0.88 & 0.69 & 1.13 & 4.92 & -0.27 & 10.1 \\
\hline $\begin{array}{l}\text { SNPs, F } \\
=11.68)\end{array}$ & 2SLS & & & & & & \\
\hline $\begin{array}{l}\text { Sleep- } \\
\text { disordered } \\
\text { breath- } \\
\text { ing }\end{array}$ & \multicolumn{2}{|c|}{ Observational1.60 } & 1.36 & 1.88 & 0.09 & -0.27 & 0.45 \\
\hline$(14$ & MR & 1.26 & 1.00 & 1.58 & 6.40 & 1.15 & 11.7 \\
\hline $\begin{array}{l}\text { SNPs, F } \\
=10.27)\end{array}$ & \multicolumn{2}{|l|}{ 2SLS } & & & & & \\
\hline $\begin{array}{l}\text { Sleep } \\
\text { quality }\end{array}$ & \multicolumn{2}{|c|}{ Observational1.01 } & 1.00 & 1.01 & -0.13 & -0.29 & 0.03 \\
\hline & MR & 1.42 & 1.06 & 1.92 & 0.94 & -4.82 & 6.70 \\
\hline $\begin{array}{l}\text { SNPs, F } \\
=7.09)\end{array}$ & 2SLS & & & & & & \\
\hline
\end{tabular}

FEV1/FVC, forced expiratory volume in $1 \mathrm{~s} /$ forced vital capacity; MR 2SLS, Mendelian randomization two-stage least square; OR, odds ratio; CI, confidence interval; SNP, single-nucleotide polymorphism. Observational models were cross-sectional logistic models which were adjusted for age, sex, parental education, and family income.

Odds ratios and beta values were estimated using the interquartile range increase of obesity-related factors.

Table 3. Hazard ratios of asthma for obesity-related factors in the longitudinal cohort

\begin{tabular}{|c|c|c|c|c|c|c|c|c|}
\hline $\begin{array}{l}\text { Risk } \\
\text { factors }\end{array}$ & $\begin{array}{l}\text { Incident } \\
\text { asthma }\end{array}$ & $\begin{array}{l}\text { Incident } \\
\text { asthma }\end{array}$ & $\begin{array}{l}\text { Incident } \\
\text { asthma }\end{array}$ & $\begin{array}{l}\text { Incident } \\
\text { atopic } \\
\text { asthma }\end{array}$ & $\begin{array}{l}\text { Incident } \\
\text { atopic } \\
\text { asthma }\end{array}$ & $\begin{array}{l}\text { Incident } \\
\text { atopic } \\
\text { asthma }\end{array}$ & $\begin{array}{l}\text { Incident } \\
\text { nonatopic } \\
\text { asthma }\end{array}$ & $\begin{array}{l}\text { Incident } \\
\text { nonatopic } \\
\text { asthma }\end{array}$ \\
\hline & HR & $95 \%$ & $95 \%$ & HR & $95 \%$ & $95 \%$ & HR & $95 \%$ \\
\hline & & CI & CI & & CI & CI & & CI \\
\hline Obesity & 1.28 & 1.05 & 1.56 & 0.62 & 0.27 & 1.44 & 1.37 & 0.98 \\
\hline $\begin{array}{l}\text { Physical } \\
\text { activ- } \\
\text { ity }\end{array}$ & 1.02 & 0.96 & 1.08 & 1.03 & 0.77 & 1.23 & 1.09 & 1.00 \\
\hline
\end{tabular}




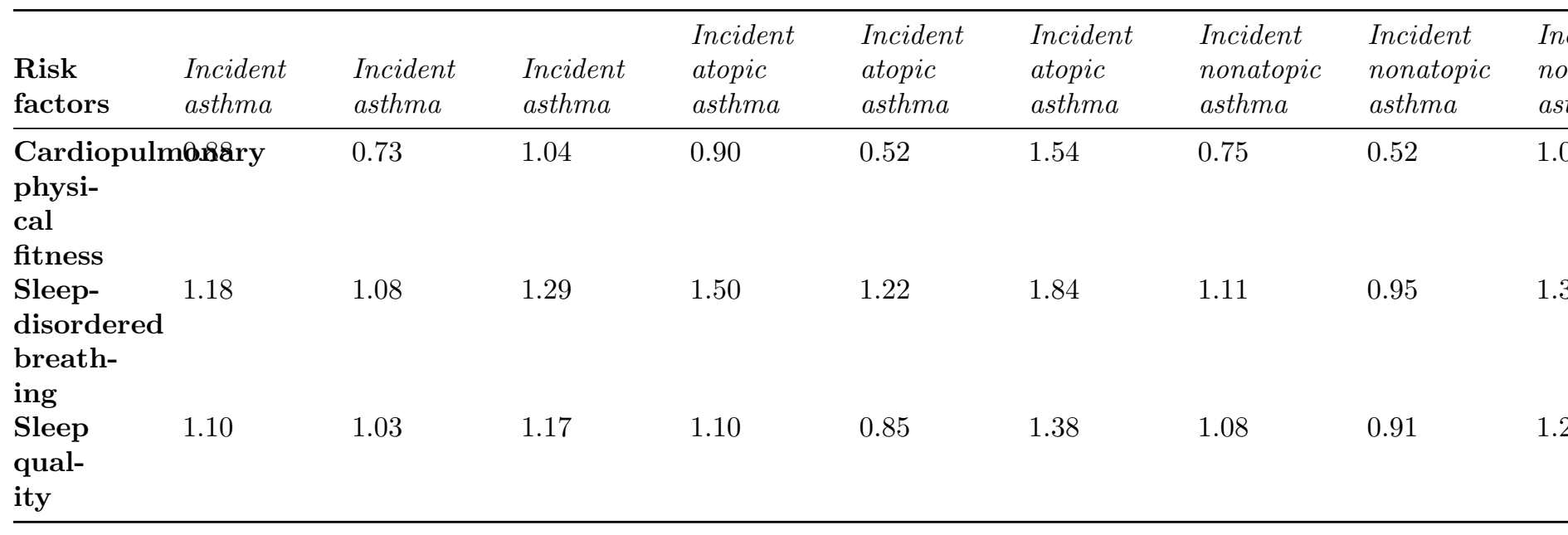

CI, confidence interval; HR, hazard ratio. Discrete-time hazard models were adjusted for age, sex, parental education, and family income.

Hazard ratios were estimated using the interquartile range increase of obesity-related factors.

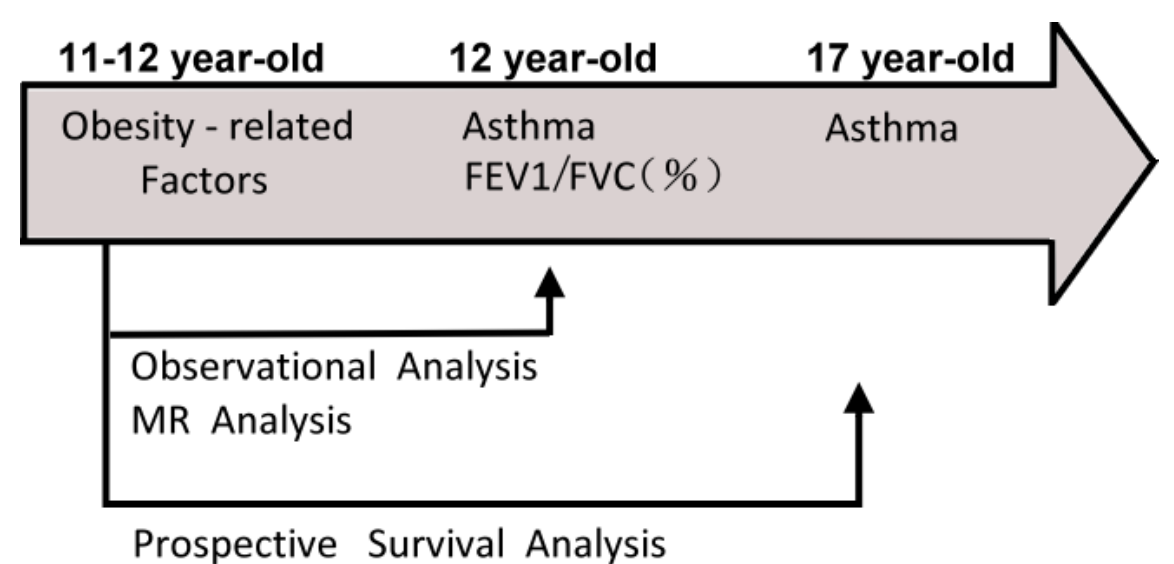




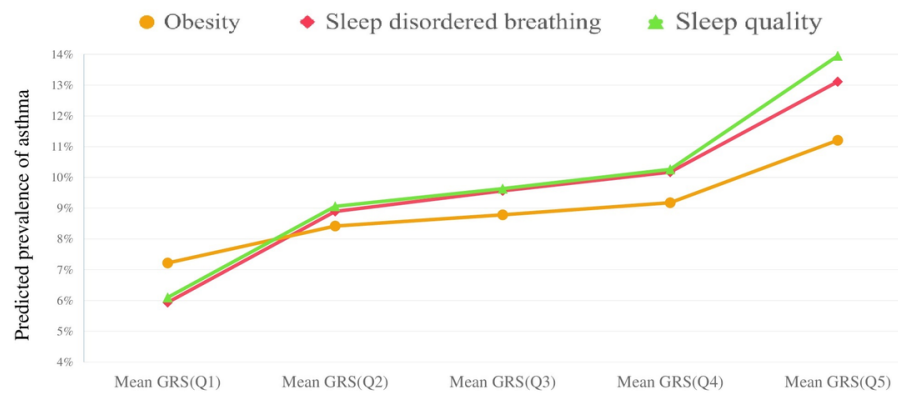

